\title{
Early readmission for congestive heart failure predicts late mortality after cardiac surgery
}

\author{
Richard Lee, MD, MBA, Natalie Homer, BS, Adin-Cristian Andrei, PhD, Edwin C. McGee, MD, \\ S. Chris Malaisrie, MD, Preeti Kansal, MD, and Patrick M. McCarthy, MD
}

\begin{abstract}
Objective: Early readmission in patients hospitalized for medical congestive heart failure is common, expensive, and associated with a worse late survival. Our objective was to compare late survival in patients' readmission for congestive heart failure with readmission for other causes in patients undergoing cardiac surgery.
\end{abstract}

\begin{abstract}
Methods: Of 3654 consecutive patients undergoing cardiac surgery at a single institution between April 2004 and June 2010, $3492(96 \%)$ were discharged from the hospital before 30 days and analyzed. Survival curves by readmission reason were compared using the log-rank test. Multivariable analyses adjusted for patient demographics, known preoperative cardiac risk factors, and surgical characteristics.

Results: The readmission rate at 30 days was 13\% (465/3492): $23 \%$ for arrhythmias/heart block, $12 \%$ for congestive heart failure, $40 \%$ for surgery related causes, $14 \%$ for infection, and $11 \%$ for noncardiac causes. Independent risk factors for readmission include age, gender, congestive heart failure, and cardiopulmonary bypass time. Eight percent (268/3492) of discharged patients died within the 6-year study: $14 \%$ in the readmission group versus $7 \%$ in the nonreadmission group $(P<.01)$. Patients who had been readmitted for congestive heart failure had worse late survivals compared with all patients who had been readmitted for causes related to their surgery.
\end{abstract}

Conclusions: Readmission within 30 days after cardiac surgery for congestive heart failure predicts late mortality. Targeted postoperative management may be warranted in patients with surgical congestive heart failure. (J Thorac Cardiovasc Surg 2012;144:671-6)

Early readmission is common in patients hospitalized for medical congestive heart failure (CHF). Estimated 30-day readmission rates approach $25 \%{ }^{1,2}$ among patients with previous heart failure hospitalization. In the elderly population, readmission rates may be as high as $45 \%{ }^{3,4}$ Frequent readmissions are associated with high personal and shared medical costs, and burden the already stressed health care system. In addition to its monetary consequence, readmission has long served as a predictor of worse patient outcomes. All-cause readmission after heart failure hospitalization has proven to be a predictor of both 1-year and total mortality in elderly outpatient populations. ${ }^{5,6}$

Although early readmission has been widely studied in the population with medical heart failure, limited data availability precluded an accurate description of the relationship between early readmission and late outcomes after cardiac surgery. The Society of Thoracic Surgeons National Database reports 30-day readmission rates within this patient population to range from $9.0 \%$ to $14.5 \%$, varying by

\footnotetext{
From the Bluhm Cardiovascular Institute, Northwestern University, Chicago, Ill. Disclosures: Authors have nothing to disclose with regard to commercial support. Received for publication Feb 3, 2012; revisions received April 9, 2012; accepted for publication May 15, 2012; available ahead of print June 19, 2012.

Address for reprints: Richard Lee, MD, MBA, 201 E Huron St, Suite 11-140,

Chicago, IL 60611 (E-mail: ricklee@nmh.org).

$0022-5223 / \$ 36.00$

Copyright (c) 2012 by The American Association for Thoracic Surgery doi:10.1016/j.jtcvs.2012.05.031
}

surgery type. ${ }^{7}$ As in medical heart failure readmission, readmission in the population who underwent cardiac surgery is expensive and believed to be associated with poorer outcomes. However, this patient population requires separate study because readmission causes and subsequent outcomes differ from those of patients with nonsurgical heart failure. Our objective was to define the impact of 30-day readmission on late survival after cardiac surgery, with specific focus on those patients' readmission for CHF.

\section{PATIENTS AND METHODS}

A total of 3654 patients underwent cardiac surgery at Northwestern Memorial Hospital between April 2004 and June 2010. This project was approved by the institutional review board at Northwestern University (institutional review board project STU000012288). Any subject refusing participation in the project was not included in this analysis. Included surgeries were aortic and mitral valve repairs and replacements, coronary artery bypass grafting $(\mathrm{CABG})$, and combined procedures. All ventricular assist device and transplant recipients were excluded from study. The remaining 3492 patients $(96 \%)$ discharged from the hospital before 30 days after surgery constitute our study population. Their institutional database records were retrospectively collected and analyzed. All readmissions to Northwestern Hospital were included from institutional records. In addition, readmissions from outside hospitals were determined by a mailed survey that inquired of any hospital admissions or procedures since discharge and included an information release form to allow our data collection from other institutions. Readmission occurrence and reason were determined and adjudicated by Northwestern University's Bluhm Cardiovascular Institute Clinical Trials Unit staff based on institutional progress notes and discharge summaries in accordance with the Society of Thoracic Surgeons database. $^{7}$ Readmission reasons were grouped into 5 categories: 


$$
\begin{aligned}
& \text { Abbreviations and Acronyms } \\
& \begin{aligned}
\text { BMI } & =\text { body mass index } \\
\text { CABG } & =\text { coronary artery bypass grafting } \\
\text { CHF } & =\text { congestive heart failure } \\
\text { CI } & =\text { confidence interval } \\
\text { OR } & =\text { odds ratio }
\end{aligned}
\end{aligned}
$$

(1) arrhythmia, (2) CHF, (3) infection (deep sternum, conduit harvest site, or pneumonia), (4) surgery-related reason (acute vascular complication, anticoagulation complication, coronary artery dysfunction, myocardial infarction, pericardial effusion or tamponade, permanent cerebrovascular accident, renal failure or transient ischemic attack, or other surgeryrelated readmission, including surgical complications such as graft failure), and (5) nonrelated reason (including gastrointestinal complications and bleeds). Mortality was ascertained by Social Security Death Index and telephone follow-up.

\section{Statistical Analyses}

The association between every baseline variable (patient demographics, preoperative variables, and surgical characteristics) and 30-day readmission status was measured using a series of univariate logistic regression models. Variables significant at a 2 -sided 5\% alpha level are shown in Table 1. Variables in Table 1 were used as the initial pool of factors to create a multivariate logistic regression model. A stepwise variable selection procedure with $10 \%$ entry/exit significance levels was performed. The resulting multivariate logistic regression model was subsequently adjusted for surgical procedure type (isolated $\mathrm{CABG}$, isolated valve, and $\mathrm{CABG}+$ valve). All significant variables in the final model are presented in Table 2. A similar approach was used to construct a multivariate model for 30-day CHF readmission. As such, variables significantly associated with 30-day CHF readmission in univariate and multivariate fashion are shown in Tables 3 and 4, respectively. All-cause long-term mortality was summarized using Kaplan-Meier survival curves. Overall survivorship comparisons between the readmission and nonreadmission groups were based on the log-rank test. A Cox regression model that adjusts for surgical procedure and all the univariate 30-day readmission predictors listed in SAS 9.2 software (SAS Inc, Cary, NC) was used to perform all statistical analyses. Statistical significance was established at the 2 -sided $5 \%$ alpha level, and no multiplicity adjustments were made.

\section{RESULTS}

Some $66 \%$ of our patient population was male (2321/ 3492 ) with a mean body mass index (BMI) of 28.0 $( \pm 5.8)$. Mean age was $63.2 \pm 13.7$ years, and $81 \%$ of surgeries were elective.

\section{Risk Factors for 30-Day All-Cause Readmission After Cardiac Surgery}

Of the 3492 patients discharged within 30 days of cardiac surgery, $465(13 \%)$ required hospital readmission within 30 days of surgery. Of these patients, $23 \%$ were readmitted for arrhythmia or heart block, $12 \%$ were readmitted for CHF, $14 \%$ were readmitted for infection, $40 \%$ were readmitted for surgery-related reasons, and $11 \%$ were readmitted for nonrelated reasons (Table 5).

Significant univariate 30-day readmission risk factors are shown in Table 1. They include age, gender, history of CHF, and New York Heart Association class III or IV. Initial length of hospital stay was also a significant predictor for early readmission: mean $7.67 \pm 4.17$ days for readmission versus $7 \pm 3.88$ days for nonreadmission (odds ratio [OR], 1.04; 95\% confidence interval $[\mathrm{CI}], 1.02-1.06 ; P<.001$ ). Independent multivariate predictors for 30-day readmission presented in Table 2 are age (OR, 1.13 per decade), female gender (OR, 1.29), history of CHF (OR, 1.29), cardiopulmonary bypass time (OR, 1.30 per hour), and readmission to the intensive care unit $(\mathrm{OR}, 1.53)$.

\section{Risk Factors for 30-Day Congestive Heart Failure Readmission After Cardiac Surgery}

Significant univariate 30-day CHF readmission risk factors are shown in Table 3 and include age, gender, history of CHF, history of atrial fibrillation, and initial length of hospital stay: median 8 (interquartile range, 6-12) days in

\begin{tabular}{|c|c|c|c|}
\hline Variable & OR & $95 \% \mathrm{CI}$ & $P$ value \\
\hline Age by decade & 1.13 & $1.05-1.22$ & .002 \\
\hline Gender, female & 1.32 & $1.07-1.62$ & .009 \\
\hline Diabetes & 1.31 & $1.03-1.67$ & .027 \\
\hline Renal failure requiring dialysis & 1.79 & $1.00-3.21$ & .049 \\
\hline Hypertension & 1.28 & $1.03-1.59$ & .026 \\
\hline Prior cardiac intervention & 1.28 & $1.04-1.57$ & .019 \\
\hline Prior valve surgery & 1.46 & $1.02-2.08$ & .041 \\
\hline $\mathrm{CHF}$ & 1.52 & $1.22-1.90$ & $<.001$ \\
\hline NYHA class III/IV & 1.34 & $1.09-1.64$ & .005 \\
\hline History of atrial fibrillation & 1.41 & $1.12-1.77$ & .003 \\
\hline Preoperative anticoagulant use & 1.33 & $1.01-1.75$ & .041 \\
\hline Preoperative aspirin use & 1.30 & $1.05-1.62$ & .018 \\
\hline Preoperative beta-blocker use & 1.33 & $1.08-1.64$ & .007 \\
\hline Preoperative antiarrhythmic use & 1.48 & $1.02-2.13$ & .037 \\
\hline LVEF by $10 \%$ & 0.92 & $0.85-0.99$ & .026 \\
\hline Coronary artery disease & 1.40 & $1.03-1.91$ & .030 \\
\hline Tricuspid insufficiency $\geq 2+$ & 1.52 & $1.15-2.02$ & .004 \\
\hline $\mathrm{CABG}$ procedure & 1.09 & $0.90-1.33$ & .385 \\
\hline Aortic valve surgery & 1.13 & $0.92-1.38$ & .236 \\
\hline Mitral valve surgery & 1.01 & $0.82-1.25$ & .921 \\
\hline Tricuspid valve surgery & 1.75 & $1.26-2.44$ & $<.001$ \\
\hline Other cardiac surgery & 1.33 & $1.09-1.61$ & .005 \\
\hline Atrial fibrillation ablation surgery & 1.40 & $1.09-1.79$ & .008 \\
\hline Cardiopulmonary bypass time (h) & 1.29 & $1.16-1.44$ & $<.001$ \\
\hline Crossclamp time (h) & 1.30 & $1.13-1.50$ & $<.001$ \\
\hline Intraoperative blood products used & 1.46 & $1.19-1.79$ & $<.001$ \\
\hline Postoperative blood products used & 1.32 & $1.08-1.61$ & .006 \\
\hline Readmission to the ICU & 1.92 & $1.29-2.85$ & .001 \\
\hline Length of stay (d) & 1.04 & $1.02-1.06$ & $<.001$ \\
\hline
\end{tabular}
CHF readmission versus 6 (interquartile range, 5-8) days in CHF nonreadmission (OR, 1.11; 95\% CI, 1.06-1.16; $P<.001)$. Independent multivariate predictors for 30-day readmission presented in Table 2 are history of CHF

TABLE 1. Univariate risk factors for 30-day readmission

$O R$, Odds ratio; $C I$, confidence interval; $C H F$, congestive heart failure; $N Y H A$, New York Heart Association; $L V E F$, left ventricular ejection fraction; $C A B G$, coronary artery bypass grafting; $I C U$, intensive care unit. 
TABLE 2. Multivariate risk factors for 30-day readmission

\begin{tabular}{lccr}
\hline \multicolumn{1}{c}{ Characteristics } & OR & $\mathbf{9 5 \%}$ CI & P value \\
\hline Age by decade & 1.13 & $1.04-1.23$ & .006 \\
Gender, female & 1.30 & $1.04-1.63$ & .019 \\
CHF & 1.31 & $1.03-1.66$ & .028 \\
Cardiopulmonary bypass time & 1.31 & $1.17-1.47$ & $<.001$ \\
Readmission to the ICU & 1.52 & $0.98-2.36$ & .059
\end{tabular}

Variables entered into the model include age; gender; diabetes; dialysis; hypertension; previous cardiac intervention or valve surgery; CHF; New York Heart Association class III/IV; history of atrial fibrillation; anticoagulant, aspirin, beta-blocker, and antiarrhythmic use; ejection fraction; coronary artery disease; tricuspid regurgitation 2+; tricuspid valve surgery; atrial fibrillation ablation surgery; cardiopulmonary bypass time; crossclamp time; intraoperative and postoperative blood products; readmission to the intensive care unit; and length of stay. $O R$, Odds ratio; $\mathrm{CI}$, confidence interval; $C H F$, congestive heart failure; $I C U$, intensive care unit.

(OR, 3.36), history of atrial fibrillation (OR, 3.16), preoperative aspirin use (OR, 2.24), and postoperative blood products $(\mathrm{OR}, 2.80)$.

\section{Overall 30-Day Readmission Predicts Late Mortality}

Eight percent (268/3492) of patients discharged within 30 days after cardiac surgery died during the 6-year study period: $14 \%$ of the readmission group and $7 \%$ of the nonreadmission group $(P<.001)$ (Figure 1$)$.

TABLE 3. Risk factors for readmission in those readmitted for congestive heart failure, univariate analysis

\begin{tabular}{|c|c|c|c|}
\hline Characteristics & OR & $95 \% \mathrm{CI}$ & $P$ value \\
\hline Age by decade & 1.33 & $1.04-1.69$ & .022 \\
\hline Dyslipidemia & 2.08 & $1.09-3.95$ & .026 \\
\hline Chronic lung disease & 2.10 & $1.06-4.15$ & .034 \\
\hline Peripheral vascular disease & 2.60 & $1.08-6.26$ & .033 \\
\hline Prior cardiac intervention & 3.22 & $1.80-5.77$ & $<.001$ \\
\hline Prior $\mathrm{CABG}$ & 3.69 & $1.79-7.58$ & $<.001$ \\
\hline Prior PCI & 2.15 & $1.06-4.35$ & .033 \\
\hline Prior MI & 2.88 & $1.47-5.65$ & .002 \\
\hline $\mathrm{CHF}$ & 3.51 & $1.92-6.44$ & $<.001$ \\
\hline NYHA class III/IV & 2.69 & $1.50-4.82$ & $<.001$ \\
\hline History of atrial fibrillation & 3.29 & $1.74-6.24$ & $<.001$ \\
\hline $\begin{array}{l}\text { Preoperative warfarin } \\
\text { (Coumadin; Bristol-Myers } \\
\text { Squibb, New York, NY) use }\end{array}$ & 3.09 & $1.31-7.27$ & .010 \\
\hline Preoperative aspirin use & 2.33 & $1.28-4.25$ & .006 \\
\hline LVEF by $10 \%$ & 0.69 & $0.56-0.84$ & $<.001$ \\
\hline Coronary artery disease & 2.27 & $1.02-5.07$ & .045 \\
\hline Left main disease & 2.94 & $1.22-7.10$ & .017 \\
\hline Tricuspid insufficiency $\geq 2+$ & 2.66 & $1.34-5.26$ & .005 \\
\hline Repeat sternotomy & 2.95 & $1.56-5.58$ & $<.001$ \\
\hline Intraoperative blood products used & 3.17 & $1.53-6.53$ & .002 \\
\hline Postoperative blood products used & 3.23 & $1.69-6.17$ & $<.001$ \\
\hline Total hours in the ICU & 1.01 & $1.00-1.01$ & .003 \\
\hline Length of stay (d) & 1.09 & $1.03-1.16$ & .002 \\
\hline
\end{tabular}

All models are adjusted for type of surgery: isolated $\mathrm{CABG}$, isolated valve, and $\mathrm{CABG}+$ valve. $O R$, Odds ratio; $C I$, confidence interval; $C A B G$, coronary artery bypass grafting; $P C I$, percutaneous coronary intervention; $M I$, myocardial infarction; $C H F$, congestive heart failure; NYHA, New York Heart Association; $L V E F$, left ventricular ejection fraction; $I C U$, intensive care unit.
TABLE 4. Multivariable analyses: Risk factors for 30-day readmission for congestive heart failure

\begin{tabular}{lccr}
\hline \multicolumn{1}{c}{ Characteristics } & OR & 95\% CI & $\boldsymbol{P}$ value \\
\hline CHF & 2.89 & $1.42-5.86$ & .003 \\
History of atrial fibrillation & 4.94 & $2.33-10.46$ & $<.001$ \\
Preoperative aspirin use & 2.31 & $1.14-4.66$ & .020 \\
LVEF by 10\% & 0.78 & $0.62-0.98$ & .034 \\
Postoperative blood products used & 2.81 & $1.42-5.57$ & .003 \\
\hline
\end{tabular}

Variables entered into the model include age, dyslipidemia, chronic lung disease, peripheral vascular disease, previous cardiac intervention, previous CABG, previous percutaneous coronary intervention, myocardial infarction, CHF, New York Heart Association class III/IV, history of atrial fibrillation, warfarin (Coumadin) and aspirin use, ejection fraction, coronary artery disease, left main disease, tricuspid regurgitation $2+$, repeat sternotomy, intraoperative and postoperative blood products, total hours in the intensive care unit, and length of stay. $O R$, Odds ratio; $C I$, confidence interval; $C H F$, congestive heart failure; $L V E F$, left ventricular ejection fraction.

After adjusting for surgical procedure type (isolated $\mathrm{CABG}$, isolated valve, and $\mathrm{CABG}+$ valve) and all other univariate predictors in Table 1, 30-day readmission remained a statistically significant predictor of late mortality (hazard ratio, $1.45 ; 95 \% \mathrm{CI}, 1.04-2.02 ; P=.028$ ).

\section{Late Mortality Rates by Readmission Reason}

Patients readmitted for CHF and noncardiac-related reasons had the highest long-term mortality rates (Table 6). Kaplan-Meier survival curves were created for the nonreadmission group and 5 readmission groups (Figure 2), and were compared using the log-rank test. Readmission for CHF was found to be associated with significantly worse late survival than nonreadmission and all other readmission causes related to surgery (log-rank, $7.60 ; P=.006)$. Patients readmitted for noncardiac-related causes had similar late survival as those readmitted for CHF (log-rank, 0.71; $P=.397$ ).

TABLE 5. Thirty-day readmission patient classification $(N=465)$

\begin{tabular}{lrc}
\hline \multicolumn{1}{c}{ Readmission reason } & N & \% \\
\hline Arrhythmia/heart block & 105 & 22.6 \\
CHF & 56 & 12.0 \\
Infection & 67 & 14.4 \\
$\quad$ Deep sternum & 13 & 2.8 \\
Conduit harvest site & 15 & 3.2 \\
Pneumonia & 39 & 8.4 \\
Surgery-related reason & 184 & 39.6 \\
Acute vascular complication & 3 & 0.7 \\
Anticoagulation complication & 8 & 1.7 \\
Coronary artery dysfunction & 1 & 0.2 \\
Myocardial infarction & 8 & 1.7 \\
Pericardial effusion or tamponade & 17 & 3.7 \\
Permanent CVA & 8 & 1.7 \\
Renal failure & 3 & 0.7 \\
TIA & 7 & 1.5 \\
Other surgery-related readmission & 129 & 27.7 \\
Nonrelated reason & 53 & 11.4 \\
Total readmissions & 465 & \\
\hline
\end{tabular}

$C H F$, Congestive heart failure; $C V A$, cerebrovascular accident; TIA, transient ischemic attack. 


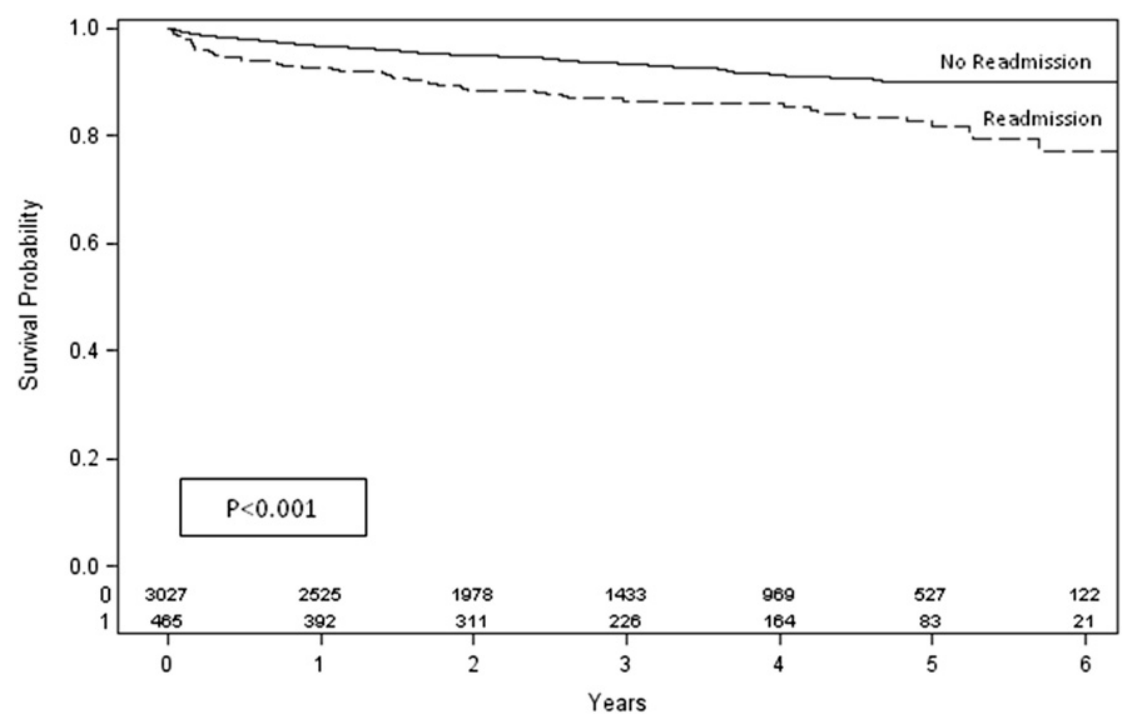

FIGURE 1. Kaplan-Meier survival curves by 30 -day readmission status $(\log$-rank, $28.9 ; P<.001)$.

Readmission for surgery-related reasons was associated with a significantly lower rate of survival compared with nonreadmission (log-rank, 11.83; $P<.001$ ). Readmission for infection was not associated with worse late survivals compared with nonreadmission (log-rank, $2.25 ; P=.134$ ). Readmission for arrhythmia was associated with significantly lower late mortality when compared with readmission for all other causes (log-rank, 11.70; $P<.001)$.

\section{DISCUSSION}

Readmission after cardiac surgery is common and associated with worse outcomes. ${ }^{1-4}$ Although the correlation between readmission and late mortality has been established in a patient population with medical CHF, limited data exist on the effect of early readmission on outcomes after cardiac surgery.

This study found older age, female gender, CHF, and increased cardiopulmonary bypass time to be significantly associated with 30-day readmission. Kumbhani and colleagues $^{8}$ reported intraoperative myocardial acidosis to also predict readmission after cardiac surgery. This variable was not analyzed in this study, but this and other surgical complications are also potential risk factors for

TABLE 6. Readmission reasons by all-cause long-term mortality

\begin{tabular}{lcc}
\hline Readmission reason & $\begin{array}{c}\text { No. deceased/no. } \\
\text { readmitted }\end{array}$ & \% Deceased \\
\hline Arrhythmia & $4 / 105$ & 3.8 \\
CHF & $16 / 56$ & 28.6 \\
Infection & $8 / 67$ & 11.9 \\
Nonrelated reason & $13 / 53$ & 24.5 \\
Surgery-related reason & $25 / 184$ & 13.6 \\
Total & $66 / 465$ & 14.2 \\
\hline
\end{tabular}

CHF, Congestive heart failure. readmission. Rockx and colleagues ${ }^{9}$ found that a BMI greater than 30 is predictive of early readmission. However, BMI was not found to be associated with higher readmission rates in this study. Because elevated BMI is linked to an increased risk of sternal wound and leg infections, of which this study had few, high BMI-related complications did not lead to a significant increase in readmissions. This study also analyzed BMI on a continuous numeric spectrum instead of grouping patients by designated BMI range as in the study by Rockx and colleagues.

Lahey and colleagues ${ }^{10}$ found that patients postoperatively discharged after 7 or more days were twice as likely to be readmitted as those discharged sooner. We also found initial length of hospital stay was significantly associated with early readmission in univariate models, but not in multivariate analysis. However, we did not isolate and further analyze the subgroup of patients with extremely long lengths of stay.

Our data suggest that not all readmissions, but specifically CHF and nonsurgery-related readmissions, are associated with reduced survival. Readmission for CHF was associated with higher mortality than all other surgeryspecific readmission and nonreadmission groups. This study identifies CHF as a risk factor for both all-cause and CHF-related early readmission. In addition, it is associated with increased all-cause long-term mortality. Thus, patients with CHF are a high-risk group for elevated hospital costs and worse outcomes. It is not surprising that the preoperative presence of $\mathrm{CHF}$ and a lower ejection fraction are associated with readmission in this group. However, these data further support the role of atrial fibrillation as a marker or cause of a sicker population within the CHF group. After further validation of these findings, to reduce both readmission and subsequent mortality, targeted 


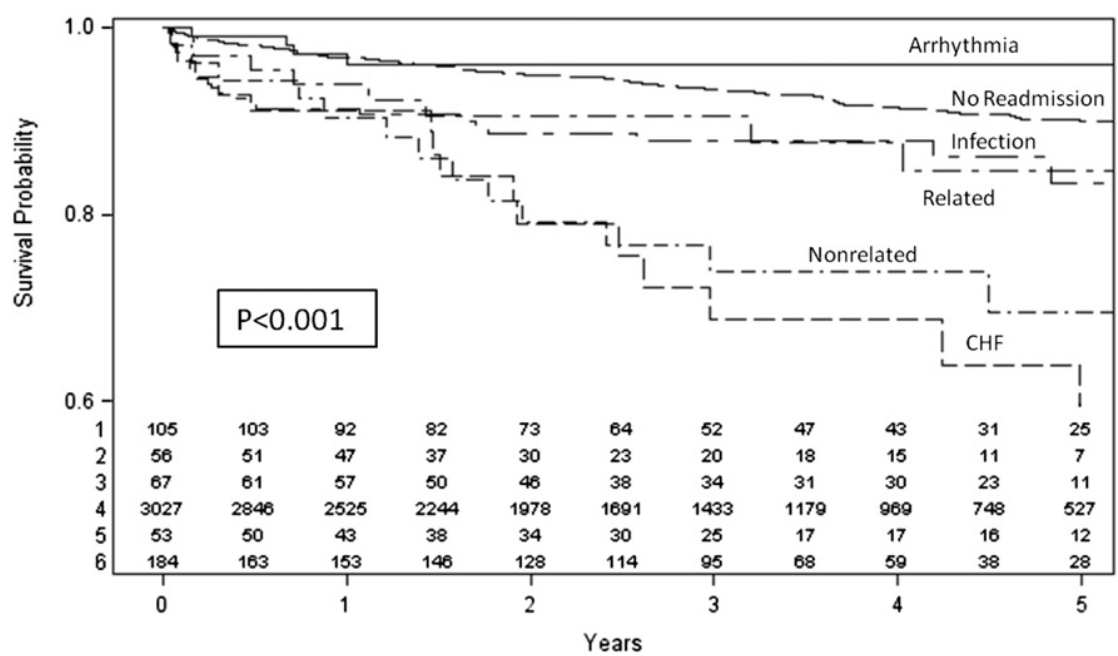

FIGURE 2. Kaplan-Meier overall survival curves by 30-day readmission status and reason. Kaplan-Meier survival curves for all readmitted types and nonreadmitted patients. $\mathrm{CHF}$, Congestive heart failure.

strategies could be developed to identify and treat high-risk patients with CHF undergoing cardiac surgery. Patients readmitted for noncardiac-related reasons, such as gastrointestinal complications, had late survival similar to those in the CHF readmission group. These patients may also benefit from targeted preventative therapies.

Patients readmitted for infection did not have different survival from those not readmitted. Filsoufi and colleagues ${ }^{11}$ alternatively found deep sternal wound infections after cardiac surgery to be associated with increased mortality. The small number of sternal wound infections in this study limited the analysis in our population. Certainly, if there were more sternal infections, there may be a different result. However, prevention remains the best method to address this problem.

Of note, readmission for arrhythmias was associated with better survival than nonreadmission. This is likely due to the group's overall healthier patient population. The presence of comorbidities also tended to be less in this group compared with other readmission and nonreadmission groups, including preoperative hypertension, diabetes, chronic obstructive pulmonary disease, and prior myocardial infarction or stroke.

This study has several limitations. It was performed at a single institution, which may reduce its widespread applicability because of differences in patient populations and care practices among treatment centers. Although extensive effort was made to identify all the readmissions in the study, some outside readmissions may not be reported. However, patient characteristics, overall readmission rate, and proportion of patients within readmission reason groups were similar to those reported in the Society of Thoracic Surgeons national database. The Society of Thoracic Surgeons national average readmission rate for all valve heart surgery, $\mathrm{CABG}$, and combined procedures is
$11.8 \%$ : $13.8 \%$ for arrhythmias, $15.8 \%$ for CHF, $20 \%$ for all infections, $15.1 \%$ for surgery-related reasons (including $2.1 \%$ myocardial infarction and $2.2 \%$ stroke), and $35.3 \%$ for nonrelated reasons, ${ }^{7}$ which is comparable to our experience.

Further, study duration is limited to 6 years and a relatively small population. To perform statistical analyses, readmission types were grouped into 5 categories. The readmission groups for infection, surgery-related reasons, and nonrelated reasons were particularly heterogeneous, with the possibility that certain subgroups directionally shifted associated trends. If a larger patient population was available for study, additional readmission groups could be analyzed to more specifically pinpoint high-risk patients.

Finally, unknown confounders are always a concern in retrospective studies. Determination of unknown causes for readmission and worse late survival are of importance because readmission is not itself a cause but a predictor of late mortality.

\section{CONCLUSIONS}

Despite the study's limitations, to the best of our knowledge, this is the first systematic study of the relationship between early readmission and late mortality in a patient population undergoing cardiac surgery. Our data suggest that not all readmission reasons incur increased mortality, but similar to the population with medical CHF, patients with surgical CHF who are readmitted postoperatively fare worse. Early identification of patients at risk for readmission and targeted postoperative management strategies may lead to improved survival.

\section{References}

1. Keenan PS, Normand SL, Lin Z, Drye EE, Bhat KR, Ross JS, et al. An administrative claims measure suitable for profiling hospital performance on the basis 
of 30-day all-cause readmission rates among patients with heart failure. Circ Cardiovasc Qual Outcomes. 2008;1:29-37.

2. Jencks SF, Williams MV, Coleman EA. Rehospitalizations among patients in the Medicare fee-for-service program. $N$ Engl J Med. 2009;360:1418-28.

3. Krumholz HM, Parent EM, Tu N, Vaccarino V, Wang Y, Radford MJ, et al. Readmission after hospitalization for congestive heart failure among Medicare beneficiaries. Arch Intern Med. 1997;157:99-104.

4. O'Connor CM, Abraham WT, Albert NM, Clare R, Gattis Stough W, Gheorghiade M, et al. Predictors of mortality after discharge in patients hospitalized with heart failure: an analysis from the Organized Program to Initiate Lifesaving Treatment in Hospitalized Patients with Heart Failure (OPTIMIZE-HF). Am Heart J. 2008; 156:662-73.

5. Pulignano G, Del Sindaco D, Tavazzi L, Lucci D, Gorini M, Leggio F, et al. Clinical features and outcomes of elderly outpatients with heart failure followed up in hospital cardiology units: data from a large nationwide cardiology database (INCHF Registry). Am Heart J. 2002;143:45-55.
6. Muntwyler J, Abetel G, Gruner C, Follath F. One-year mortality among unselected outpatients with heart failure. Eur Heart J. 2002;23:1861-6.

7. Society of Thoracic Surgeons National Database. Available at: http://www.sts. org/national-database. Accessed May 21, 2012.

8. Kumbhani DJ, Healey NA, Thatte HS, Birjiniuk V, Crittenden MD, Treanor PR, et al. Intraoperative myocardial acidosis as a risk for hospital readmission after cardiac surgery. Am J Surg. 2009;198:373-80.

9. Rockx MA, Fox SA, Stitt LW, Lehnhardt KR, McKenzie FN, Quantz MA, et al. Is obesity a predictor of mortality, morbidity and readmission after cardiac surgery? Can J Surg. 2004;47:34-8.

10. Lahey SJ, Campos CT, Jennings B, Pawlow P, Strokes T, Levitsky S. Hospital readmission after cardiac surgery. Does "fast track" cardiac surgery result in cost saving or cost shifting? Circulation. 1998;98(19 Suppl):II35-40.

11. Filsoufi F, Castillo JG, Rahmanian PB, Broumand SR, Silvay G, Carpentier A, et al. Epidemiology of deep sternal wound infection in cardiac surgery. J Cardiothorac Vasc Anesth. 2009;23:488-94. 